Int. J. Electrochem. Sci., 14 (2019) $1580-1590$

International Journal of

ELECTROCHEMICAL

SCIENCE

www.electrochemsci.org

\title{
A Hybrid Si@C@CNT@C Anode by Anchoring Silicon Nanoparticles onto CNT for Enhancing Performance in Lithium Ion Battery
}

\author{
Gang Fang ${ }^{1,2}$, Xiaolong Deng ${ }^{3}$, Jizhao Zou ${ }^{1 *}$, Xierong Zeng ${ }^{1,2,4}$ \\ ${ }^{1}$ Shenzhen Key Laboratory of Special Functional Materials \& Shenzhen Engineering Laboratory for \\ Advance Technology of Ceramics, College of Materials Science and Engineering, Shenzhen \\ University, Shenzen, 518060, PR China. \\ ${ }^{2}$ Key Laboratory of Optoelectronic Devices and Systems of Ministry of Education and Guangdong \\ Province, College of Optoelectronic Engineering, Shenzhen University, Shenzhen, 518060, P.R. China \\ ${ }^{3}$ GuoGuang Electric Company Ltd, No. 8 Jinghu Road, Xinhua Town, Huadu Region, Guangzhou, \\ 510800, P. R. China \\ ${ }^{4}$ Guangdong JANUS Intelligent Group Corporation Limited, Dongguan, 523878, China \\ *E-mail: zoujizhao@ szu.edu.cn
}

doi: $10.20964 / 2019.02 .73$

Received: 13 October 2018 / Accepted: 11 December 2018 / Published: 5 January 2019

\begin{abstract}
We develop a facile method to anchor commercially available silicon nanoparticles (SiNPs) onto carbon nanotube (CNT) and fabricate the SiNPs and CNT based composite (denoted as Si@C@CNT@C) through a living polymerization reaction combined with a carbonization process. Si@C@CNT@C possesses a structure in which carbon coated SiNPs are anchored onto CNT linked by a carbon layer to enhance electron transfer. Anchoring structure helps to restrain SiNPs aggregating, and carbon layer as well as CNT network facilitates buffering the volume variation and stabilizing the SEI film. Hence, as an anode material for lithium ion batteries, Si@C@CNT@C demonstrates an improved initial discharge specific capacity $\left(\sim 4162 \mathrm{mAhg}^{-1}\right)$ and coulombic efficiency $(84 \%)$ as well as much enhanced rate performance.
\end{abstract}

Keywords: Silicon nanoparticle; Carbon nanotube; Anchor effect; Anode; Lithium ion battery

\section{$\underline{\text { FULL TEXT }}$}

(C) 2019 The Authors. Published by ESG (www.electrochemsci.org). This article is an open access article distributed under the terms and conditions of the Creative Commons Attribution license (http://creativecommons.org/licenses/by/4.0/). 